\section{In a sphere of their own}

Tokuyama Dental's latest creation, Omnichroma colourless composite, relies totally on optical mimicry allowing to match exactly to surrounding tooth colouring. Omnichroma matches virtually every tooth.

Often, little consideration is given to the filler of a composite material, other than the ratio of filler to resin. That is until Tokuyama's research showed that the size and shape of composite filler particles are vital to creating optimal success with the material. Most brands of composites contain irregularly shaped and sized fillers. This makes polishing and shade-blending a difficult challenge.

Tokuyama is the only composite manufacturer to use patented spherical filler particles within their composite materials, each variant utilising spherical particles of different diameters to maximise their optical and physical properties for the desired indication.

These unique spherical fillers are made through the sol-gel process, a method of producing glass and ceramic materials discovered in the mid-eighteenth century. In the 1990s, sol-gel research gained pace with over 35,000 papers published, coinciding with Tokuyama's development of their dental composite materials.

The sol-gel process can be controlled to produce perfect spheres, uniform in their composition. Further control of the process allows Tokuyama to determine the exact dimensions of the spheres, enhancing the ability of the material to reflect light in a uniform manner, creating composite materials with excellent shade matching and aesthetic properties.

The spherical particles and the structure they form within the composite lead to refraction and reflection of light that mimics the opalescence of natural tooth. Higher diffusion and light refraction results in a material that will offer the ability for excellent aesthetic

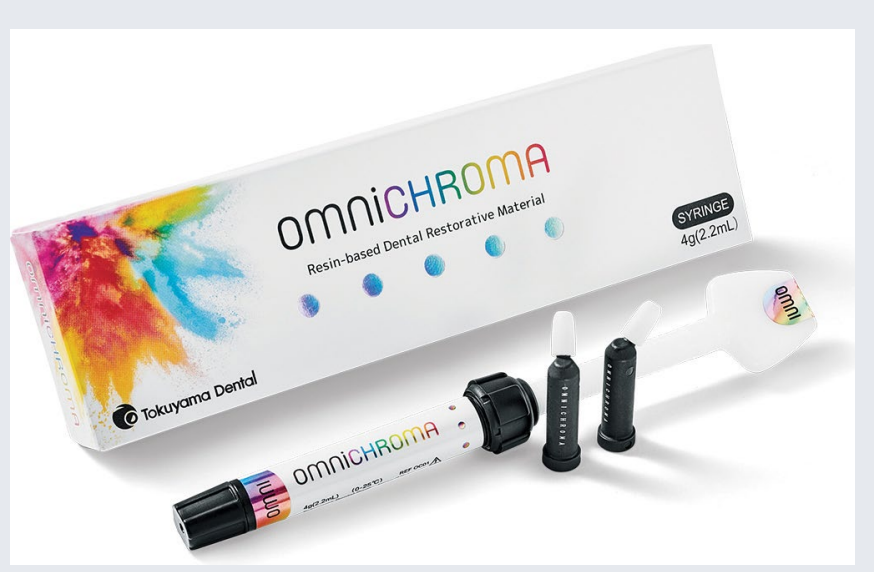

blending with adjacent tooth structure.

By carefully adapting the sol-gel process to create particles of exactly $260 \mathrm{~nm}$ diameter, it was discovered that for the first time colour could be achieved without addition of a pigment. The unique filler composition deliberately induces the phenomenon of structural colour to combine with the reflected colour of the surrounding tooth. The result: Omnichroma.

The spherical filler particles also improve the gloss and lustre of the finished restoration. Spherically filled composites reached $90 \%$ glossiness after only one minute of polishing, compared to a prominent composite material with irregular filler size that achieved $70 \%$ glossiness in the same period of time. Additionally, the material with a spherical filler retains its gloss and lustre longer due to the enhanced wear characteristics.

For more information about the complete Tokuyama range, including Omnichroma, contact your local Trycare Representative, call 01274885544 or visit www.trycare.co.uk/omnichroma.

\title{
A smart, compact, affordable unit
}

Acteon UK has taken panoramic 2D and 3D CBCT to another level, a new elevation to be more precise, namely the wall. XMIND PRIME is a smart, compact, affordable unit. It is also wall-mounted, ideal for any practice lacking the space for a floor standing unit.

Where lack of available space and cost has previously prevented a practice purchasing a panoramic digital imaging unit, this is no longer an issue.

There is no correlation between size and capability. The $2 \mathrm{D}$ option offers a full set of high-quality images to meet all clinical applications in general dentistry and patient positioning is simple with the easy to use interface. The 3D-CBCT option offers all of this plus a minimum voxel size of $87.5 \mu \mathrm{m}$ for greater detail and even more applications that cater for the needs of dental specialists and GDPs. Implant planning is easy and a report can be created in one minute, impression and model scanning is possible and the diagnostic capabilities are vast, thanks to our Acteon Imaging Suite Software (AIS).

AIS allows users to view all images captured from ACTEON imaging devices (and other manufacturers). Integrating with all leading practice management software it facilitates diagnosis with intuitive image manipulation, simplified implant planning, open architecture and advanced functionality.

Acteon UK specialises in the manufacture and supply of minimally

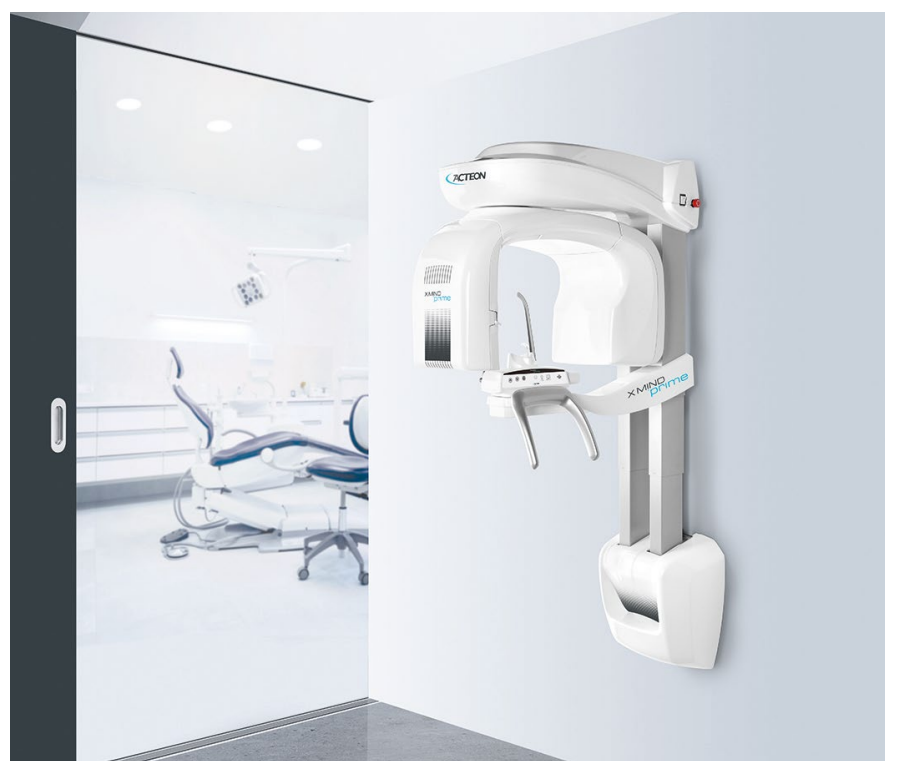

invasive innovative technologies for dentists and dental professionals to diagnose, treat and educate patients efficiently and are leading the fight against periodontal disease.

For more information call 08000389840 (ext 1), email info.uk@ acteongroup.com or visit acteongroup.com/uk-en. 